\title{
Prise en charge de patients hémophiles en Chirurgie buccale : est-il possible de les traiter en cabinet de ville?
}

\section{Strube N}

CHU, Nantes, France

dr.strube@hotmail.fr

L'hémophilie est une maladie rare mais grave. Chez ces patients, certains actes de chirurgie dentaire peuvent se compliquer d'hémorragies importantes. Le diagnostic des maladies hémorragiques implique la connaissance de la physiologie de l’hémostase. (Grehco 2004; Gomez Moreno G et al. 2005)

La conduite à tenir et la structure d'accueil peuvent varier en fonction du type d'hémophilie, de la sévérité du déficit en facteurs anti-hémophiliques, du risque hémorragique lié au geste (Piot et al. 2003 ; Société Francophone de Médecine Buccale et de Chirurgie Buccale Recommandations 2005).

Dans la majorité des cas la prise en charge hospitalière est nécessaire ; toutefois certains patients (déficit mineur répondant favorablement à la vasopressine) peuvent être traités en ville. La collaboration avec l'hématologue demeure indispensable et la prise de décision doit être collégiale.

L'attitude actuelle limite le plus possible la quantité de facteurs injectés aussi bien pour diminuer le risque de développement d'anticorps dirigés contre le facteur VIII ou le facteur IX que pour leur coût (Goudemand 2001).

L'utilisation de vasopressine en voie veineuse (Minirin ${ }^{\circledR}$ ) ou en spray nasal (Octim ${ }^{\circledR}$ ) constitue un progrès incontestable qui autorise de plus en plus d'actes chirurgicaux sans transfusion de facteurs et en ambulatoire (Hillman et al. 2007, Negrier et al. 2009).

Nous illustrerons grâce à des cas cliniques les possibilités et les limites des traitements chirurgicaux et implantaires en ambulatoire chez les hémophiles. 